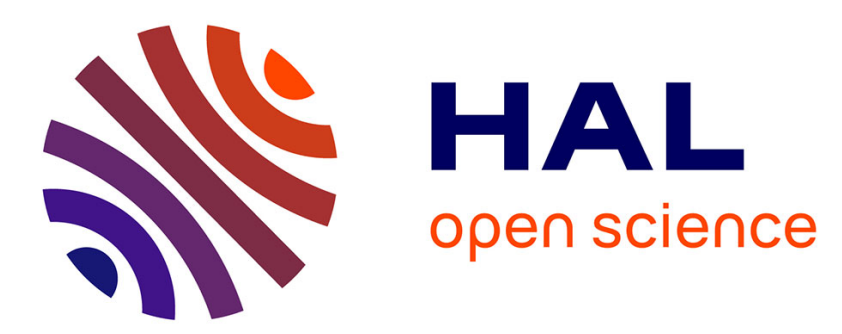

\title{
Factors beyond enolase 2 and mitochondrial lysyl-tRNA synthetase precursor are required for tRNA import into yeast mitochondria
}

M. Baleva, M. Meyer, N. Entelis, I. Tarassov, P. Kamenski, B. Masquida

\section{- To cite this version:}

M. Baleva, M. Meyer, N. Entelis, I. Tarassov, P. Kamenski, et al.. Factors beyond enolase 2 and mitochondrial lysyl-tRNA synthetase precursor are required for tRNA import into yeast mitochondria. / Biochemistry, 2017, 82 (11), pp.1324-1335. 10.1134/S0006297917110104 . hal-03470212

\author{
HAL Id: hal-03470212 \\ https://hal.science/hal-03470212
}

Submitted on 8 Dec 2021

HAL is a multi-disciplinary open access archive for the deposit and dissemination of scientific research documents, whether they are published or not. The documents may come from teaching and research institutions in France or abroad, or from public or private research centers.
L'archive ouverte pluridisciplinaire HAL, est destinée au dépôt et à la diffusion de documents scientifiques de niveau recherche, publiés ou non, émanant des établissements d'enseignement et de recherche français ou étrangers, des laboratoires publics ou privés. 
Factors beyond enolase 2 and mitochondrial lysine tRNA synthetase precursor are required for tRNA import in yeast mitochondria

Mariia Baleva ${ }^{1,2}$, Mélanie Meyer ${ }^{3}$, Nina Entelis ${ }^{1}$, Ivan Tarassov ${ }^{1}$, Piotr Kamenski ${ }^{2}$ \& Benoît Masquida $^{1 *}$

${ }^{1}$ GMGM, CNRS - University of Strasbourg, UMR 7156, 4 allée Konrad Roentgen, 67081, Strasbourg, France

${ }^{2}$ Molecular Biology Department, Faculty of Biology, M.V. Lomonosov Moscow State University, 1/12 Leninskie Gory, 119991 Moscow, Russian Federation

${ }^{3}$ Present Address: IGBMC, CNRS - University of Strasbourg, UMR 7104, 1 rue Laurent Fries, 67404 Illkirch, France

*To whom correspondance should be addressed : b.masquida@unistra.fr 


\begin{abstract}
In yeast, the import of tRNA ${ }^{\text {Lys }}$ with CUU anticodon (tRK1) relies on a complex mechanism where interaction with enolase2 (Eno2p) dictates a deep conformational change of the tRNA. This event is believed to mask the tRNA from the cytosolic translational machinery in order to be re-directed towards the mitochondria. Once in the vicinity of the mitochondrial outer membrane, the precursor of the mitochondrial lysyl-tRNA-synthetase (preMSK) takes over enolase to carry the tRNA within the mitochondrial matrix where it is supposed to participate in translation following correct refolding. Biochemical data herein focus on the role of enolase. They show that despite the inability of Eno2p alone to form a complex with tRK1, mitochondrial import can be recapitulated in vitro using various fractions of yeast extracts sharing Eno2p as one of the main components. Taken together, our data suggest the existence of an Eno2p-containing protein complex involved in RNA mitochondrial import.
\end{abstract}

Keywords: RNA mitochondrial import; RNA chaperone; tRNA-enolase interaction; enolase; moonlighting protein 


\section{Introduction}

Occurrences of mitochondrial RNA import have been characterized throughout the eukaryotic tree of life. Genetic analysis of the mitochondrial DNA organization of highly divergent eukaryotes point to tRNAs as potentially representing the largest RNA family imported into mitochondria. In plasmodia, the mitochondrial DNA encodes no tRNA nor rRNA, but three proteins necessary to the oxidative phosphorylation [1]. In trypanosomatids, the mitochondrial DNA also does not encode any tRNA [2] and tRNAs are massively imported. Moreover, in some species of cnidarians like the Lophelia pertusa coral, the mitochondrial DNA encodes only two tRNA genes, and seven proteins [3], [4]. Massive tRNA import is thus expected to support mitochondrial translation in these organisms, although their RNA import mechanism has been less studied than in plants [5].

Our laboratory has demonstrated that tRNA mitochondrial import occurs naturally in yeast [6], [7] and that yeast cytosolic tRNAs derivatives can be imported in mitochondria from cultured human cells [8], even though their mitochondrial DNA encode complete sets of tRNA. In addition, the human 5S ribosomal RNA is also imported into human mitochondria [9], [10] although its function once imported remains elusive. In yeast, the import of tRK1 (tRNA ${ }^{\text {Lys }}$ with anticodon CUU) rescue mitochondrial translation at non-permissive temperature when the mitochondrial tRNA ${ }^{\text {Lys }}$ (tRK3) is hypo-modified and becomes inefficient at decoding rare AAG codons [11]. Moreover, yeast tRNA derivatives can fortuitously suppress mutations leading to MELAS [12] or MERRF [13] syndroms, functionally replacing host mitochondrial tRNA(Leu) or tRNA(Lys), respectively. These studies suggest participation of imported cytosolic type molecules in the mitochondrial translation process. 
Studies of the mechanisms on which RNA import rely have stressed the strong implication of RNA targets refolding by molecuar chaperonnes. Either considering tRK1 or 5S, the mechanisms look very much alike with a first step of RNA recognition by a cytosolic protein trigerring RNA refolding and escape from cytosolic pathways of a small fraction of the RNA pool ( $\sim 5 \%)$. The first protein carries its RNA freight to the mitochondrial surface where it is transferred to a second carrier facilitating the translocation to the mitochondrial matrix. Although this late stage is believed to rely on the VDAC and/or TOM/TIM channels due to evidence for plants, no undisputed molecular mechanism was proposed for yeast or human mitochondria. In the case of tRK1, the glycolytic enzyme enolase is first involved as a carrier, and the precursor of the lysyl-tRNA synthetase (preMSK) appears during the second step (Figure 1). The 5S rRNA is first taken in charge by the precursor of the mitochondrial ribosomal protein L18 (MRPL18) and then by rhodanese, an enzyme involved in cyanide detoxication. Our recent studies have shown that the three human enolases $(\alpha, \beta, \gamma)$ promote mitochondrial import of tRK1 as does enolase 2 in yeast [14]. However in our efforts to characterize the complex between tRK1 and enolase 2, we have realized that additional factors seem to be required. The present study summarizes original, although preliminary, biochemical evidence, which point to potential candidates.

\section{Material and Methods}

\section{In vitro transcription of tRNAs}

Plasmid DNA template were typically obtained from XL1blue cells using the QIAGEN Plasmid Maxi Kit, and linearized with MvaI (BstNI) (Fermentas, Thermo Fisher Scientific). The construct of tRK1 and tRK2 are preceded by a hammerhead ribozyme preserving the loop-loop tertiary interaction between stems I and II in order to produce a tRK1 with wild 
type 5' and 3' sequences following design principles reported in [15]. tRNA transcription reactions were incubated during $4 \mathrm{~h}$ at $37^{\circ} \mathrm{C}$ under the following conditions: $40 \mathrm{mM}$ Tris- $\mathrm{HCl}$ (pH 8.0), 5 mM DTT, $0.01 \%$ Triton X-100, 1 mM spermidine, 5 mM ATP, 5 mM CTP, 5 mM UTP, $5 \mathrm{mM}$ GTP, $22 \mathrm{mM} \mathrm{MgCl}$, $60 \mu \mathrm{g} / \mathrm{mL}$ purified recombinant T7 RNAP (homemade), 1 units $/ \mathrm{mL}$, E. coli inorganic pyrophosphatase, and $50 \mu \mathrm{g} / \mathrm{mL}$ linearized plasmid DNA template. RNA transcripts were purified by $8-10 \%$ denaturing PAGE [16].

\section{Recombinant enolase 2 expression and purification}

The wild-type yeast enolase 2 (Eno2p) with an N-terminal $\mathrm{His}_{6}$-tag was prepared from Escherichia coli strain BL21 carrying the plasmid pET3a. The bacteria were grown at $37^{\circ} \mathrm{C}$ in $\mathrm{LB}$ media containing $0.1 \mathrm{mg} / \mathrm{ml}$ ampicilline until the $\mathrm{OD}_{600}$ reached 0.6 , and protein expression was induced with $0.5 \mathrm{mM}$ IPTG for $2-3 \mathrm{~h}$ at $30^{\circ} \mathrm{C}$. The cells were harvested by centrifugation and resuspended in $\mathrm{NP}$ buffer $\left(50 \mathrm{mM} \mathrm{NaH}_{2} \mathrm{PO}_{4}, 300 \mathrm{mM} \mathrm{NaCl} \mathrm{pH}\right.$ 8.0) supplemented with Complete EDTA-free protease inhibitor tablets (Roche). Cells were incubated in the presence of lysozyme $(1 \mathrm{mg} / \mathrm{ml}), 30 \mathrm{~min}$ on ice and lysed by sonication. Cell debris were collected by centrifugation at $20,000 \mathrm{~g}$ for $1 \mathrm{~h}$ at $4^{\circ} \mathrm{C}$. The supernatant was incubated overnight at $4^{\circ} \mathrm{C}$ with Ni-NTA resin (Qiagen) in presence of $10 \mathrm{mM}$ imidazole. The resin was then washed with NPI-buffer containing 10, 20 or $30 \mathrm{mM}$ imidazole. The bound Eno2p was eluted from the resin with NPI-buffer containing imidazole concentrations increasing from $100 \mathrm{mM}$ to $200 \mathrm{mM}$. The resulting protein sample was dialyzed at $4^{\circ} \mathrm{C}$ against a buffer containing $50 \mathrm{mM}$ HEPES-KOH pH 7,5, $100 \mathrm{mM} \mathrm{KCl,} 1 \mathrm{mM} \mathrm{MgCl}$. The concentration of protein was determined by measuring absorbance at $280 \mathrm{~nm}(\mathrm{E} 1 \%=8,95)$. The purity of the protein was verified by SDS-PAGE.

\section{Purification of yeast enolase 2}

Native Eno2p was purified from $\triangle e n o 1$ yeast cells (ENO1 gene was deleted in W303 yeast strain by KanMX4 cassette replacement) from cells at the end of the exponential phase. Yeast cells were grown in liquid YPD medium (1\% yeast extract, $2 \%$ peptone, $2 \%$ glucose/dextrose) 
supplemented with $0.2 \mathrm{mg} / \mathrm{ml}$ of $\mathrm{G} 418$ at $30^{\circ} \mathrm{C}$ in a shaking incubator (Infors HT Multitron, $250 \mathrm{rpm}$ ) for $18 \mathrm{~h}$. The cells were harvested by centrifugation and washed with pre-chilled milliQ water.

Disruption of cells was performed with fastprep using glass beads in BB buffer $(0,6 \mathrm{M}$ Sorbitol, $10 \mathrm{mM}$ Tris-HCl, $\mathrm{pH}$ 7,5, $1 \mathrm{mM}$ EDTA and proteases inhibitors cocktail). Cell debris were collected by centrifugation at $40000 \mathrm{~g}$ for $30 \mathrm{~min}$ at $4{ }^{\circ} \mathrm{C}$, and $67 \%$ saturation of ammonium sulfate was achieved by addition of solid ammonium sulfate. After centrifugation at $40000 \mathrm{~g}$ at $4^{\circ} \mathrm{C}$ for $30 \mathrm{~min}$, the supernatant was $100 \%$ saturated and centrifuged again at $40000 \mathrm{~g}$ at $4^{\circ} \mathrm{C}$ for $30 \mathrm{~min}$. The supernatant was discarded and the pellet dissolved in $20 \mathrm{mM}$ Tris- $\mathrm{HCl}$ buffer, $\mathrm{pH} 8.3$, containing $5 \mathrm{mM} \mathrm{MgSO}_{4}, 1 \mathrm{mM}$ EDTA; dialyzed overnight against the same buffer and applied to a mono-Q column (HiTrap Q XL, GE Healthcare) equilibrated with $10 \mathrm{mM}$ Tris- $\mathrm{HCl}$ buffer, $\mathrm{pH} 8.3$, containing $5 \mathrm{mM} \mathrm{MgSO}_{4}, 1 \mathrm{mM}$ EDTA. Proteins were fractionated with a linear $\mathrm{NaCl}$ gradient $(0-1 \mathrm{M})$. Eno2p-enriched fractions were applied to a Superdex-200 Increase 10/300 GL column (GE Healthcare) equilibrated with 50 mM HEPES$\mathrm{KOH}, \mathrm{pH} 7.5,150 \mathrm{mM} \mathrm{Kcl,} 5 \mathrm{mM} \mathrm{MgSO} 4$ and $1 \mathrm{mM}$ EDTA. A final step using hydroxyapatite chromatography (CHT5-1 BioRad) was used to eliminate nucleases. At each purification step, protein-containing fractions were probed by dot-blot analysis using an antienolase antibody.

\section{Isothermal titration microcalorimetry (ITC)}

ITC experiments were conducted on a MicroCal ITC200 (GE Healthcare). Prior to the experiment, the protein and RNA samples were dialyzed against the same buffer $(25 \mathrm{mM}$ HEPES-KOH (pH 7.5); 100 mM KCl; $1 \mathrm{mM} \mathrm{MgCl}_{2} ; 0,1$ mM EDTA). Data treatment was performed with the software Origin 7.0. Typically $300 \mu \mathrm{L}$ of a solution of Eno2p at $30 \mu \mathrm{M}$ was used to fill up the main adiabatic cell, and the syringe was loaded with a solution containing $300 \mu \mathrm{M}$ RNA refolded under the same conditions as for EMSA experiments. 


\section{Electrophoretic Mobility Shift Assay (EMSA)}

T7 transcripts of tRK1 were 5' end-labeled with $\gamma$ - ${ }^{32} \mathrm{P}$-ATP using T4

polynucleotide kinase (Promega). The labeled RNA was denatured at $92^{\circ} \mathrm{C}$ and then slowly cooled down to room temperature in presence of $4 \mathrm{mM}$ EDTA. For gel-shifts, appropriate amounts of proteins and labeled tRK1 transcripts were mixed in $10 \mu \mathrm{L}$ of a buffer containing $25 \mathrm{mM}$ HEPES-KOH (pH 7.5); $100 \mathrm{mM} \mathrm{KCl} ; 1 \mathrm{mM} \mathrm{MgCl}_{2}, 0.1 \mathrm{mM}$ EDTA and incubated for $20 \mathrm{~min}$ at $30^{\circ} \mathrm{C}$. RNA-protein complexes were separated from free RNA by native $8 \%$ PAGE containing 5\% glycerol in $0.5 x$ TBE buffer ( $\mathrm{pH} 8.3)$.

\section{In vitro mitochondrial import assay}

Purified mitochondria from yeast YPH499 incubated with radio-labeled RNA were added to purified proteins or protein fractions prepared in import buffer ( $0.6 \mathrm{M}$ sorbitol, 20 mM HEPES-KOH (pH 6.8), 20 mM KCl, 2,5 mM MgCl 2,1 mM ATP, 5 mM DTT, 0,5 $\mathrm{mM}$ phosphoenol pyruvate and 4 units of pyruvate kinase). For a standard in vitro assay, $50000 \mathrm{cpm}$ of labelled RNA were added per $0.1 \mathrm{ml}$ of the reaction mixture containing $0.1 \mathrm{mg}$ of mitochondria as deduced from the amount of mitochondrial protein as determined by Bradford assay. After $15-20$ min incubation at $30^{\circ} \mathrm{C}, 50 \mu \mathrm{g} / \mathrm{ml}$ of RNase A (Sigma) was added and the reaction was further incubated for $10 \mathrm{~min}$ on ice to digest all unimported RNAs. The mitochondria were washed three times with a buffer containing $0.6 \mathrm{M}$ sorbitol, 10 mM HEPES-KOH (pH 6.7) and 4 mM EDTA. Then mitochondria were pelleted by centrifugation and resuspended in $100 \mu \mathrm{l}$ of TRIzol reagent (Termo Fisher Scientific). RNA was precipitated with ethanol and the samples fractionated by $8 \%$ denaturing PAGE $(8 \mathrm{M}$ urea), followed by quantification with a Typhoon-Trio scanner using the Image Quant-Tools software (GE Healthcare). The amount of the imported RNA was determined by comparison of the band density of the protected full-sized RNA isolated from the mitochondria after the import assay to an aliquot representing $2-5 \%$ of the RNA input. 


\section{Western blot}

For immunoblotting, proteins were separated on SDS-PAGE and transferred to a nitrocellulose membrane. Goat antibodies directed against enolase were used (Molecular Probes, C-19: sc-7455, Santa Cruz Biotechnology). Western-blots were conducted according to standard procedures.

\section{Purification of S100 fraction and heparin chromatography}

The initial protein sample represents an extract of wild-type cells. An overnight $1 \mathrm{~L}$ culture of yeast W303 was centrifuged, washed twice with ice cold water, resuspended in 10 mM HEPES-KOH (pH 6.8), 50 mM KCl, 1 mM EDTA, 5 mM DTT, $10 \%$ glycerol, Complete protease inhibitor cocktail (Roche). Cells were lyzed by shaking in the presence of glass beads (FastPrep, MP Biomedicals). Cell debris were pelleted by low (2000g) speed centrifugation. The supernatant was centrifuged at $100,000 \mathrm{~g}$ for $30 \mathrm{~min}$ at $4^{\circ} \mathrm{C}$. The $\mathrm{S} 100$ fraction was dialyzed against $20 \mathrm{mM}$ HEPES-KOH (pH 6,8), $10 \mathrm{mM} \mathrm{NaCl} ; 0,5 \mathrm{mM}$ PMSF, 1 mM DTT and 10\% glycerol, and applied to a heparin-sepharose column (GE Healthcare) equilibrated with the same buffer. Proteins were eluted by a step gradient of sodium chloride $(0,1 ; 0,2 ; 0,5$ and $1 \mathrm{M})$. The pooled fractions were dialyzed against $20 \mathrm{mM}$ HEPES, $\mathrm{pH}$ ?, $1 \mathrm{mM}$ DTT, $10 \mathrm{mM} \mathrm{KCl}$ and 50\% glycerol including protease inhibitor cocktail and stored at $-20^{\circ} \mathrm{C}$.

\section{Results}

\section{Improving the purity of recombinant enolase 2 abolishes interaction with tRK1} transcripts

The His-tagged recombinant enolases (wild-type, H373F) used in our biochemical assays were purified by affinity chromatography using Ni-NTA resin. Resulting samples contained fairly pure Eno2p, although some contaminants were still detected by SDS-PAGE 
(Figure 2A). Since solving the structure of the cytosolic import complex of tRK1 may be precluded by the presence of the contaminating proteins, we decided to further improve purification by addition of chromatographic steps (Figure 2B, C). Ni-NTA samples proved to be active in electro-mobility shift assays (EMSA) (Figure 2D), suggesting that tRK1 could form a complex with recombinant Eno2p.

Hydroxyapatite chromatography under native conditions indeed led to a highly pure protein sample. Yet, the propensity of the pure recombinant protein to interact and retard tRK1 was abolished (Figure 2D). The results of these experiments suggest that some contaminants may be required to promote the interaction between tRK1 and Eno2p. Since enolases from yeast and E. coli present $\sim 42 \%$ of identity [17], it is also likely that some proteins from E. coli may be efficient in binding the recombinant Eno2p, and furthermore tRK1. This possibility is strengthened by the fact that very tiny amounts of RNA are used in EMSA, thus requiring small amounts of putative interactant proteins. Moreover, EMSA performed on larger amounts of unlabeled RNA failed to detect a visible mobility shift of tRK1. Nevertheless, we could observe that proteins from E. coli (BL21 Rosetta with empty pET-3 plasmids) prepared as the His-tagged Eno2p also bound tRK1 in EMSA yielding a complex of same size as with Eno2p smaples, indicating that proteins different from the recombinant Eno2p could also interact with tRK1 (Figure 3). However we could not rule out whether E. coli enolase could replace Eno2p on this occasion.

We turned towards isothermal titration microcalorimetry (ITC) to determine whether pure Eno2p could interact with tRK1 transcripts. ITC is a very sensitive and suitable method to determine whether molecules interact in solution in an unbiased experimental setup and do not require any labelling of the studied molecules. However ITC requires much larger amounts of material than EMSA, which decreases the contribution of contaminanting proteins to the signal as opposed to EMSA. In a classical ITC experiment, a concentrated sample 
containing the ligand is added step by step to a large volume of a solution containing the receptor. At each step, the solution under continuous stirring is incubated untill the mix reaches equilibrium as monitored by the stabilization of the heat transfer. In our case, a concentrated solution of tRK1 was added step by step to a concentrated solution of Eno2p. Strikingly, no release or absorption of heat could be measured beside the very small heat variations due to mixing of the two solutions, indicating that tRK1 transcripts and Eno2p do not interact. These data confirmed abolition of complex formation with a pure Eno2p sample as observed in EMSA.

\section{Samples enriched with either recombinant or wild-type Eno2p direct in vitro import and form a detectable RNA-protein complex \\ The inability of recombinant Eno2p to interact even transiently with tRK1}

transcripts in vitro suggested that additional factors may favor tRNA mitochondrial import. In order to investigate on this point and to confirm the role of Eno2p, the ability to promote tRK1 mitochondrial import of Eno2p-enriched protein samples obtained from yeast extracts was evaluated. Wild-type Eno2p-enriched samples were obtained by tag-less purification from yeast W303 $\Delta e n o 1$ strain, a possibility due to the natural abundance of Eno2p. Enolases 1 and 2 abundance in yeast represents $6.2 \%$ and $3.6 \%$ of the total protein mass, respectively [18]. A $\Delta$ enolstrain was used to avoid interference of Eno1p with tRK1. Protein patterns of individual fractions were checked by SDS-PAGE and western blots were performed to assess the presence of Eno2p (Figure 4). Results of in vitro import experiments of tRK1 into isolated yeast mitochondria confirmed that Eno2p significantly increases import efficiency as compared to the very low level observed with preMSK alone (Figure 5). The fraction of $E$. coli proteins yielded some background comparable to preMSK, indicating that unspecific interactions of this fraction with tRK1 in EMSA experiments do not increase import efficiency in vitro. 
We wanted to check whether changing the protein composition of Eno2p-enriched fractions may affect RNA mitochondrial import. In order to narrow down the number of protein candidates that may participate in the tRNA mitochondrial import process, two strategies were undertaken. The first one consisted in serial fractionation of yeast extracts using increasing ammonium sulfate concentrations and the second one consisted in performing affinity chromatography on heparin matrix. Ammonium sulfate fractionated fractions were characterized for their content in Eno2p by western blot (Figure 6A, B) and further assayed in mitochondrial in vitro import. Only fractions containing Eno2p showed import directing capabilities (Figure 6C). Heparin chromatography permitted to collect a flow-through fraction (HP-0) containing the majority of the Eno2p pool (Figure 7). HP-0 was active in both EMSA and in mitochondrial in vitro import assays (Figure 8).

As for the recombinant Eno2p sample, we noted that yeast wild-type Eno2penriched samples underwent gradual loss of import directing capabilities after hydroxyapatite chromatography (Figure 5). As a control, import capabilities of the second cytosolic lysine tRNA isoacceptor (tRK2 with UUU anticodon) of the Eno2p-enriched fractions were evaluated. This is corroborated by the additional observation that the yeast Eno2p-enriched fractions discriminate better between tRK1 and tRK2 transcripts than recombinant Eno2p accompanied by bacterial contaminants (Figure 6D). These results were in agreement with those on recombinant Eno2p and indicate that the participation of other factors is required.

\section{Discussion}

The motivation of the present study was to characterize the interaction between Eno2p and tRK1 transcripts in order to conduct structural studies. Eno2p has been identified as a necessary factor for the initial step of tRK1 mitochondrial import in yeast [19]. Recombinant Eno2p samples obtained through nickel affinity chromatography successfully 
retardated tRK1 transcripts in electromobility shift assays (EMSA). Nevertheless, further purification steps using hydroxyapatite chromatography completely abolished tRK1 retardation. Thus, the experiments conducted herein demonstrate the absence of a direct interaction between Eno2p and tRK1 transcripts in vitro even by means of the very sensitive method that is isothermal titration microcalorimetry. The absence of interaction between pure enolase and tRK1 transcripts leads to the conclusion that additional factors should be at play. This observation is supported by the fact that Eno2p, with a fairly low isoelectric point of $\sim 5.7$, is not adapted to interact with heavily negatively charged molecules such as RNAs. Most RNA binding proteins present large positively charged electrostatic surfaces and isoelectric point usually beyond 8.0. Eno2p thus appears as a necessary, but not sufficient factor to direct tRK1 mitochondrial import. It could be argued that the absence of chemical modifications in tRK1 in vitro synthesized transcripts may prevent the two molecules to interact, although the observation that the transcripts can be sucessfully imported into mitochondria by Eno2p-enriched fractions indicates the opposite situation. Yet, the absence of chemical modifications could in principle affect import specificity, since tRK2 transcripts can also be imported in vitro, albeit not in vivo [20].

Our study indicates that fractions enriched either with recombinant or yeastpurified Eno2p are able to direct mitochondrial import in agreement with previously published results [19]. In all cases, it appears that contaminants favor this phenomenon, whether they originate from E. coli or yeast proteins. Enolases from these organisms share a high level of identity (42\%) [17], which may partially rationalize why recombinant Eno2p may benefit from $E$. coli factor(s) to carry out mitochondrial import. Since Eno2p is a very abundant cytosolic protein and that only $\sim 5 \%$ of the tRK1 pool follows the mitochondrial import route in vivo, proteins that are scarce may be involved in this mechanism. Consequently, it is possible to hypothesize that the amount of tRK1 import complex could be 
controlled by a limiting protein cofactor, which may also serve to regulate the process under stress conditions. It results from this assumption that $E$. coli contaminants participating in the process should be fairly rare molecules, hence difficult to characterize. These arguments explain why an interaction between a picomolar amount of labelled tRK1 can be detected by EMSA, while ITC, which requires micromolar amounts is less perturbed by the presence of contaminants. In spite of these evidence, we cannot completely rule out that tRK1 transcripts may be retardated by other $E$. coli proteins. A recent example describes an interaction artefact between bacterial non-coding RNAs and Hfq, which was enriched by Ni-NTA purification due to the presence of several histidine residues in the Hfq hexamere, further leading to spurious mobility shift of the target non-coding RNAs [21]. Nevertheless, E. coli proteins obtained following the recombinant Eno2p purification protocol were able to retardate tRK1 transcripts (EMSA), but did not show significant ability to promote mitochondrial import in vitro in the presence of preMSK.

The present study demonstrates that Eno2p alone is not capable of interacting with tRK1 transcripts in vitro, or to direct in vitro mitochondrial import. The data are not in contradiction with previously published ones since fractions not containing Eno2p could not direct import, and vice-versa. Nonetheless some fractions deprived of Eno2p could interact with tRK1 as detected by EMSA. Cellular unknown factors adding to Eno2p are thus necessary to direct import. The involved unidentified bacterial factors may also share high sequence identities with yeast proteins necessary to the yeast Eno2p action. Their identification will rely on further studies based on mass-spectrometry and sequence homology analysis between the E. coli and yeast proteins.

\section{Acknowledgements}

The authors thank Eric Ennifar and Cyrielle Silva Da Veiga for help with ITC measurements and data interpretation. This research is supported by the Centre National de la Recherche 
Scientifique (LABEX ANR-11-LABX-0057_MITOCROSS) and the University of

Strasbourg. MB is supported by the Russian Foundation for Basic Research (Grant 16-3400978) and the Government of the Russian Federation (State Assignment AAAA-A16116021660073-5) and French Ambassy in Moscow (Russian Federation).

\section{References}

[1] A. Sharma and A. Sharma, "Plasmodium falciparum mitochondria import tRNAs along with an active phenylalanyl-tRNA synthetase," Biochemical Journal, vol. 465, no. 3 , pp. 459-469, Feb. 2015.

[2] F. Tschopp, F. Charrière, and A. Schneider, "In vivo study in Trypanosoma brucei links mitochondrial transfer RNA import to mitochondrial protein import.," EMBO reports, vol. 12, no. 8, pp. 825-32, 2011.

[3] A. Emblem, B. O. Karlsen, J. Evertsen, D. J. Miller, T. Moum, and S. D. Johansen, "Mitogenome polymorphism in a single branch sample revealed by SOLiD deep sequencing of the Lophelia pertusa coral genome," Gene, vol. 506, no. 2, pp. 344-349, 2012.

[4] A. Emblem, B. O. Karlsen, J. Evertsen, and S. D. Johansen, "Mitogenome rearrangement in the cold-water scleractinian coral Lophelia pertusa (Cnidaria, Anthozoa) involves a long-term evolving group I intron," Mol Phylogenet Evol, vol. 61, no. 2, pp. 495-503, 2011.

[5] T. Salinas, A. M. Duchêne, and L. Maréchal-Drouard, "Recent advances in tRNA mitochondrial import," Trends in Biochemical Sciences, vol. 33, no. 7, pp. 320-329, 2008.

[6] R. P. Martin, J. M. Schneller, A. J. Stahl, and G. Dirheimer, "Import of nuclear deoxyribonucleic acid coded lysine-accepting transfer ribonucleic acid (anticodon C-UU) into yeast mitochondria," Biochemistry, vol. 18, no. 21, pp. 4600-4605, 1979. 
[7] O. A. Kolesnikova, N. S. Entelis, H. Mireau, T. D. Fox, R. P. Martin, and I. A. Tarassov, "Suppression of mutations in mitochondrial DNA by tRNAs imported from the cytoplasm," Science, vol. 289, no. 5486, pp. 1931-1933, 2000.

[8] N. S. Entelis, O. A. Kolesnikova, S. Dogan, R. P. Martin, and I. A. Tarassov, "5 S rRNA and tRNA import into human mitochondria. Comparison of in vitro requirements," J Biol Chem, vol. 276, no. 49, pp. 45642-45653, 2001.

[9] A. Smirnov, C. Comte, A. M. Mager-Heckel, V. Addis, I. A. Krasheninnikov, R. P. Martin, N. Entelis, and I. Tarassov, "Mitochondrial enzyme rhodanese is essential for 5 S ribosomal RNA import into human mitochondria," J Biol Chem, vol. 285, no. 40, pp. 30792-30803, 2010.

[10] A. Smirnov, N. Entelis, R. P. Martin, and I. Tarassov, "Biological significance of 5S rRNA import into human mitochondria: role of ribosomal protein MRP-L18," Genes Dev, vol. 25, no. 12, pp. 1289-1305, 2011.

[11] P. Kamenski, O. Kolesnikova, V. Jubenot, N. Entelis, I. A. Krasheninnikov, R. P. Martin, and I. Tarassov, "Evidence for an adaptation mechanism of mitochondrial translation via tRNA import from the cytosol," Mol Cell, vol. 26, no. 5, pp. 625-637, 2007.

[12] O. Z. Karicheva, O. A. Kolesnikova, T. Schirtz, M. Y. Vysokikh, A. M. Mager-Heckel, A. Lombes, A. Boucheham, I. A. Krasheninnikov, R. P. Martin, N. Entelis, and I. Tarassov, "Correction of the consequences of mitochondrial $3243 \mathrm{~A}>\mathrm{G}$ mutation in the MT-TL1 gene causing the MELAS syndrome by tRNA import into mitochondria," Nucleic Acids Res, vol. 39, no. 18, pp. 8173-8186, 2011.

[13] O. A. Kolesnikova, N. S. Entelis, C. Jacquin-Becker, F. Goltzene, Z. M. ChrzanowskaLightowlers, R. N. Lightowlers, R. P. Martin, and I. Tarassov, "Nuclear DNA-encoded tRNAs targeted into mitochondria can rescue a mitochondrial DNA mutation 
associated with the MERRF syndrome in cultured human cells," Hum Mol Genet, vol. 13, no. 20, pp. 2519-2534, 2004.

[14] M. Baleva, A. Gowher, P. Kamenski, I. Tarassov, N. Entelis, and B. Masquida, "A Moonlighting Human Protein Is Involved in Mitochondrial Import of tRNA," International Journal of Molecular Sciences, vol. 16, no. 5, pp. 9354-9367, 2015.

[15] M. Meyer and B. Masquida, “Cis-Acting 5' Hammerhead Ribozyme Optimization for In Vitro Transcription of Highly Structured RNAs," Methods Mol Biol, vol. 1086, pp. $21-40,2014$.

[16] M. Meyer and B. Masquida, "Polyacrylamide Gel Electrophoresis for Purification of Large Amounts of RNA,” Methods Mol Biol, vol. 1320, pp. 59-65, 2016.

[17] V. Pancholi, "Multifunctional alpha-enolase: its role in diseases," Cell Mol Life Sci, vol. 58, no. 7, pp. 902-920, 2001.

[18] P. Lu, C. Vogel, R. Wang, X. Yao, and E. M. Marcotte, "Absolute protein expression profiling estimates the relative contributions of transcriptional and translational regulation," Nat Biotechnol, vol. 25, no. 1, pp. 117-124, 2007.

[19] N. Entelis, I. Brandina, P. Kamenski, I. A. Krasheninnikov, R. P. Martin, and I. Tarassov, "A glycolytic enzyme, enolase, is recruited as a cofactor of tRNA targeting toward mitochondria in Saccharomyces cerevisiae," Genes Dev, vol. 20, no. 12, pp. 1609-1620, 2006.

[20] H. A. Kazakova, N. S. Entelis, R. P. Martin, and I. A. Tarassov, "The aminoacceptor stem of the yeast tRNA(Lys) contains determinants of mitochondrial import selectivity," FEBS Lett, vol. 442, no. 2-3, pp. 193-197, 1999.

[21] T. Milojevic, E. Sonnleitner, A. Romeo, K. Djinović-Carugo, and U. Bläsi, "False positive RNA binding activities after Ni-affinity purification from Escherichia coli,” RNA Biology, vol. 10, no. 6, pp. 1066-1069, 2014. 



\section{Figure legends}

Figure 1: Scheme of the mechanism of mitochondrial import of tRK1, the yeast lysine isoacceptor tRNA with an CUU anticodon sequence undertakes refolding through interaction with proteins including Eno2p. This complex shuttles tRK1 to the mitochondrial surface where it interacts with preMSK, which proceeds to import properly through a process that is not fully understood nor characterized in yeast or human cells.

Figure 2: (A) SDS-PAGE patterns of recombinant protein of yeast enolase $2(\sim 49 \mathrm{kDa})$ purified by Ni-NTA resin. Concentrations of imidazole in the elution buffer are indicated. (B) SDS-PAGE of fractions obtained by hydroxyapatite chromatography (C). (D) Analysis of RNA-protein interactions by electromobility shift assay (EMSA). The autoradiograph of native PAGE-separations of labeled tRK1 in the presence of eno2p show the consequences of hydroxyapatite chromatography purification. Triangles indicate increasing concentrations of Eno2p.

Figure 3: Import test with recombinant Eno2p.

Figure 4: Purification procedure of the wild-type Eno2p from yeast extract.

Figure 5: Import test with wild-type Eno2p from yeast extract.

Figure 2: (A) SDS-PAGE of Ni-NTA purified recombinant protein of yeast enolase 2 (MW of eno2p $\approx 49 \mathrm{kDa}$ ). Concentrations of imidazole in the elution buffer are indicated. (B) Analysis of RNA-protein interactions by EMSA. The native PAGE of labeled tRK1 in the presence of Eno2p shows how tRK1 binding is altered following hydroxyapatite 
chromatography. Triangles indicate increasing concentrations of Eno2p (1.2 and $3 \mu \mathrm{M})$. (C) Purification of Ni-NTA purified recombinant Eno2p samples on hydroxyapatite columns (blue: $\mathrm{OD}_{280}$; green: salt gradient from 0 to $1 \mathrm{M}$; grey, conductivity). (D) SDS-PAGE of fractions obtained by hydroxyapatite chromatography of Ni-NTA purified eno2p;

Figure 3: (A) SDS-PAGE of Ni-NTA fractionation of total E. coli proteins showing that a significant amount of proteins can be retained at fairly high concentrations of imidazole. (B) EMSA analysis of interaction between tRK1 and E. coli proteins indicate their contribution to an EMSA observable signal.

Figure 4: (A) Strategy of proteins fractionation. Total cell lysate was treated by ammonium sulfate precipitation followed by several steps of chromatography. (B) Typical chromotograms obtained by gel filtration or hydroxyapatite are shown (blue: $\mathrm{OD}_{280}$ ). (C) SDS-PAGE of collected fractions followed by western blot (D) allows to identify Eno2penroched fractions. The fraction that was used for additional step of hydroxyapatite chromatography is in frame.

Figure 5: In vitro import analyses of tagless yeast enolase 2. tRK1 input corresponds to the fraction that was used in mitochondrial in vitro import assay. Two negative control experiments are performed, without mitochondria or without IDPs (import directing proteins). A positive control is realized using preMSK, which directs import, albeit weakly. Other lanes correspond to test of specific fractions; E. coli proteins eluted at $80 \mathrm{mM}$ imidazole; recombinant his-tagged Eno2p; enriched-Eno2p fractions prior to or after hydroxyapatite chromatography.

Figure 6: (A) SDS-PAGE of samples resulting from ammonium sulfate fractionation (the concentration of ammonium sulfate is indicated) and Western immunodetection of Eno2p (B). 
(C) In vitro import assay of tRK1 transcripts by ammonium sulfate fractions compared with native yeast Eno2p. (D) In vitro import of tRK2 transcript (tRNA ${ }^{\text {Lys }}$ with anticodon UUU).

Figure 7: (A) Chromotography fractionation of crude yeast proteins. (A) SDS-PAGE of protein fractions obtained by heparin-chromatography of yeast proteins extract (S100), $10 \mu \mathrm{g}$ of proteins per lane (Bradford assay). (B) Western blot analysis of fractions obtained by heparin with antibodies against enolase. Recombinant yeast enolase 2 was used as positive control.

Figure 8: Analysis of tRK1-protein interaction. (A) ${ }^{32} \mathrm{P}$-end labeled tRK1 were incubated in the presence of fractions obtained by heparin chromatography (HP, $5 \mu \mathrm{g}$ of proteins per each reaction). The number indicates the eluant salt concentration at which the corresponding fractions were obtained. Each reaction was fractionated on native 8\% PAGE (A) or on denaturing 8\% PAGE (B). This strategy aimed at vizualizing tRK1-protein complexes and tRK1 stability in the conditions of the reaction. (C) The ability of the protein fractions used in EMSA was evaluated by in vitro mitochondrial import assay of ${ }^{32} \mathrm{P}$-end labeled tRK1 transcripts into isolated yeast mitochondria. 
A
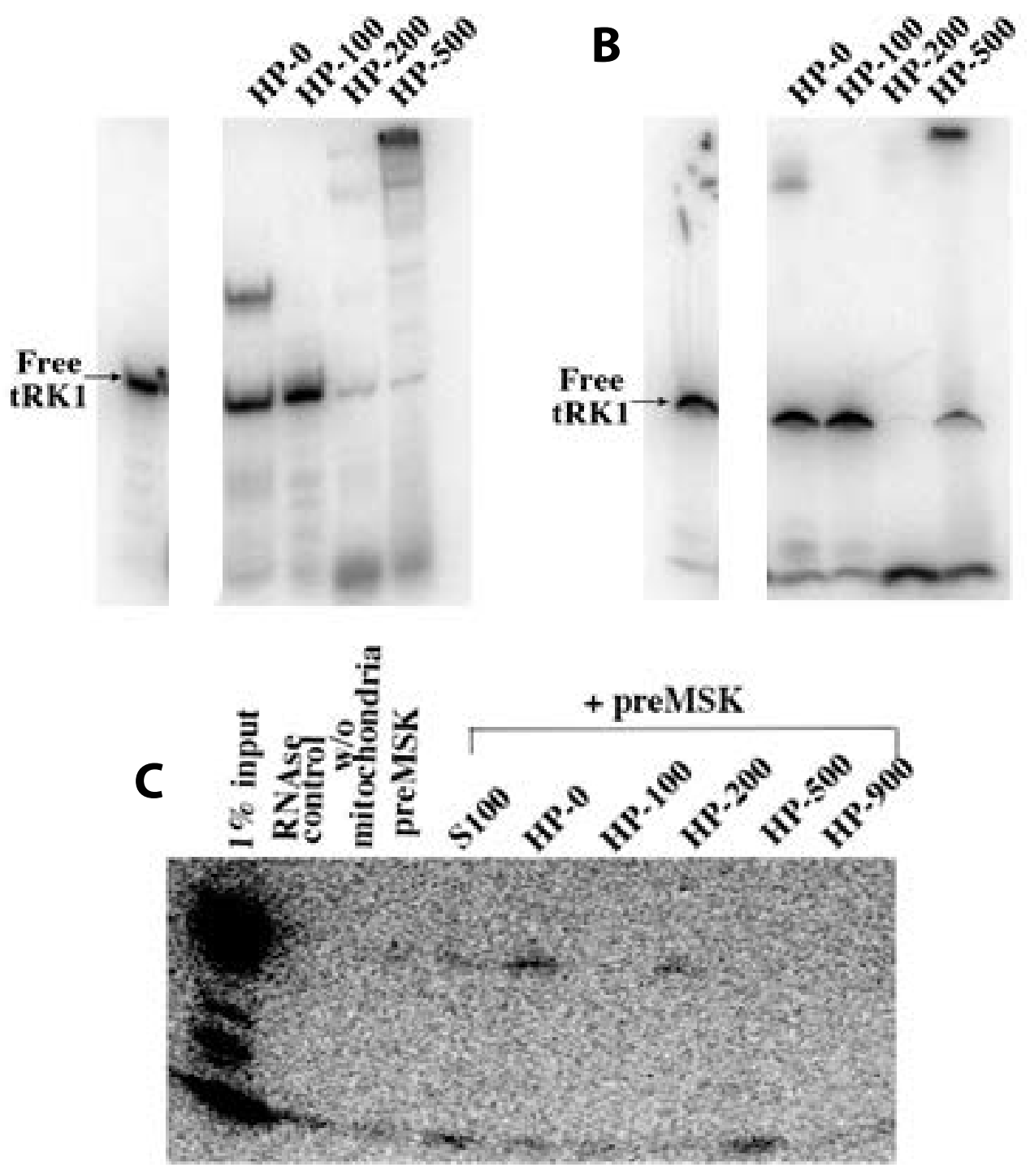


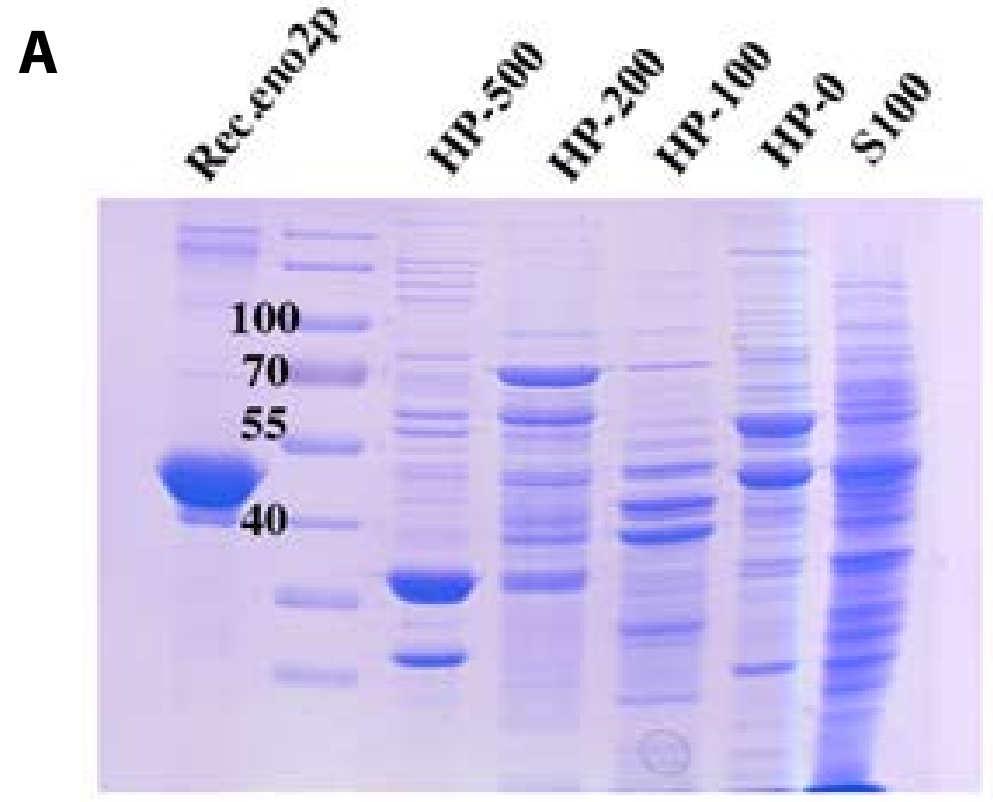

B

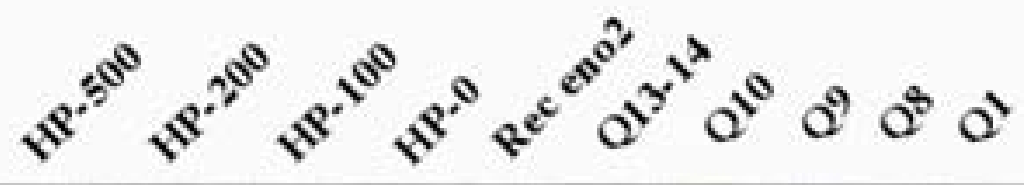

Western immunoblot with anti-enolase 
A

\section{B}

anti-enolase

antibodies
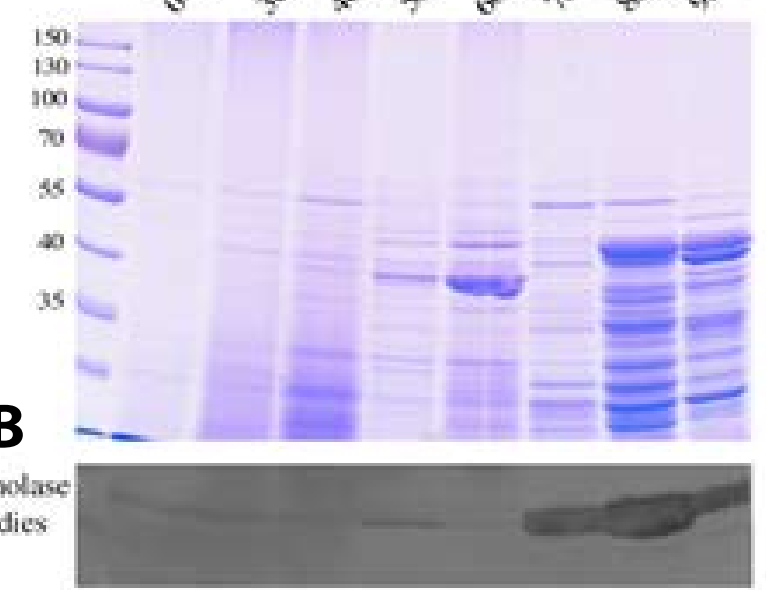

C

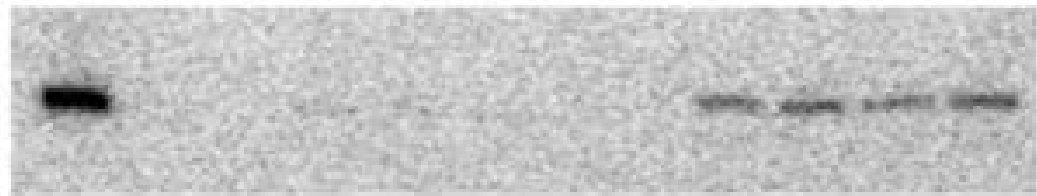

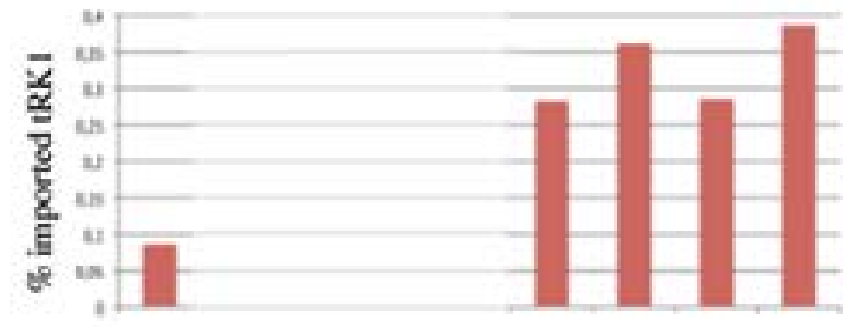

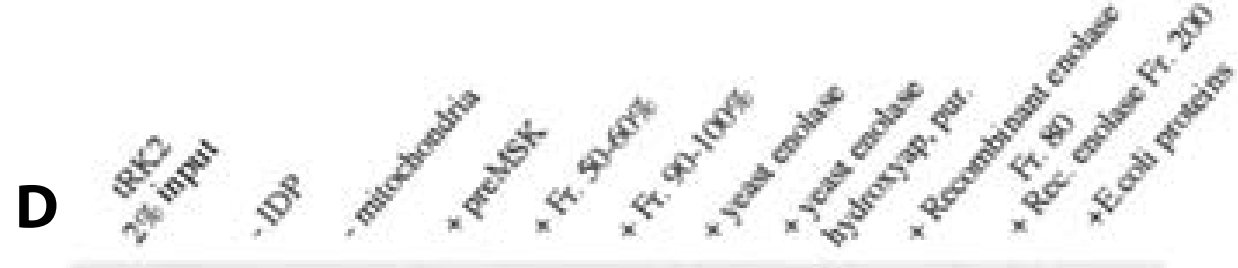
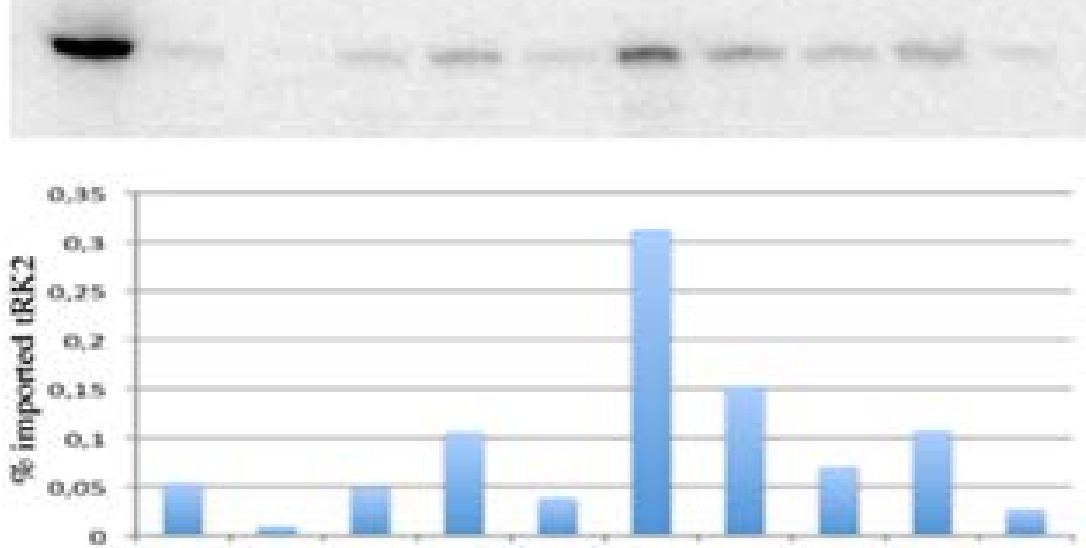

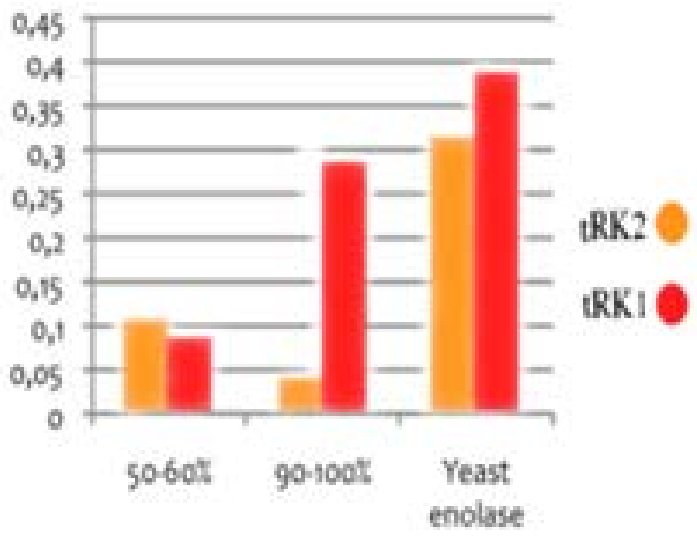



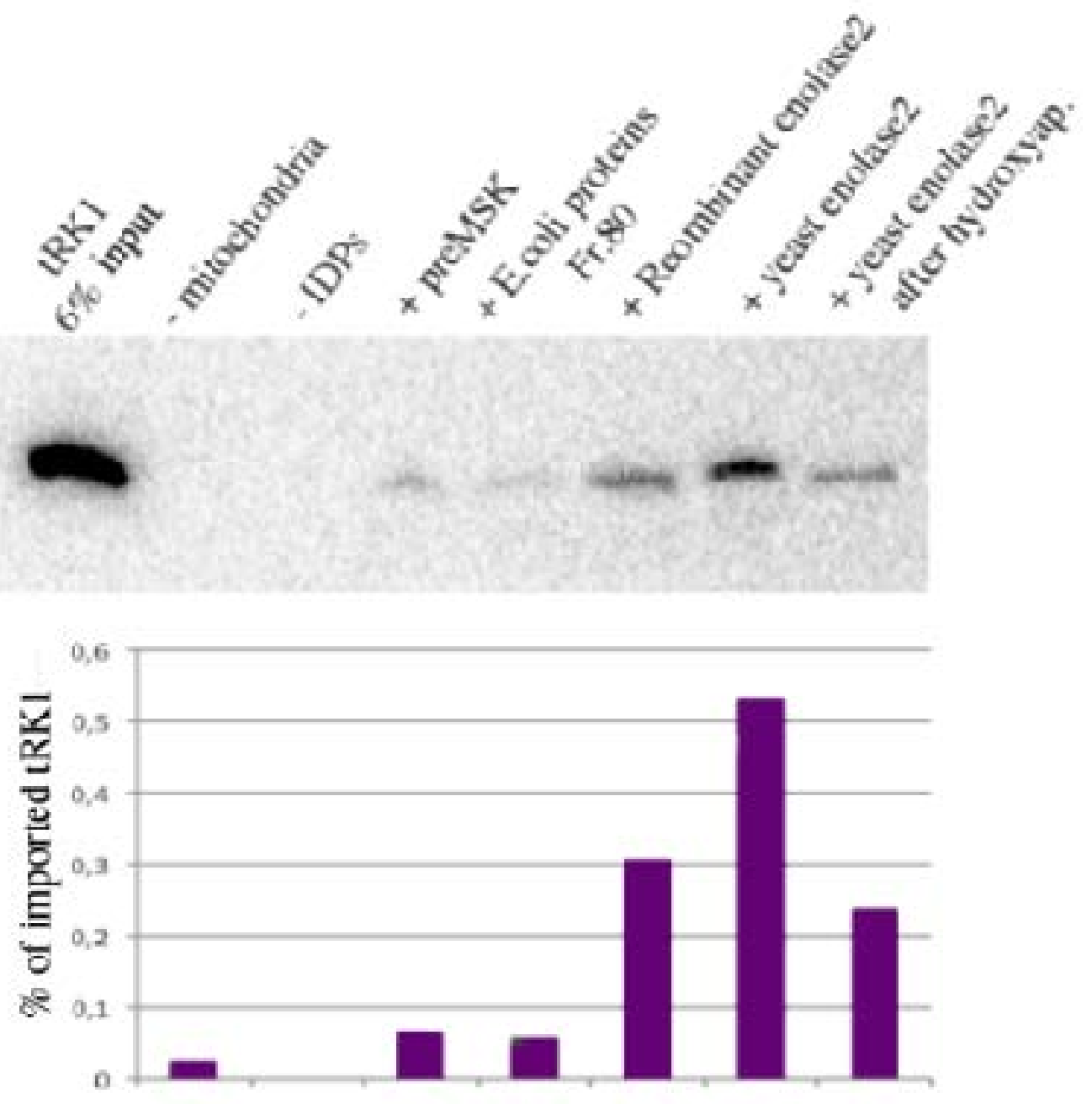


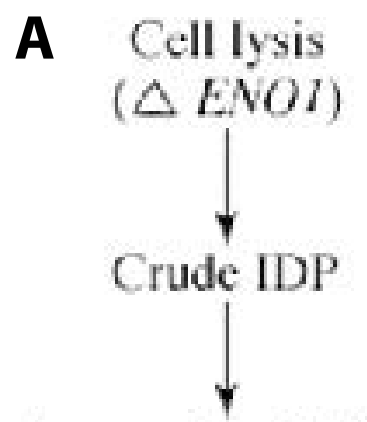

Ammonium Sulfate precipitation

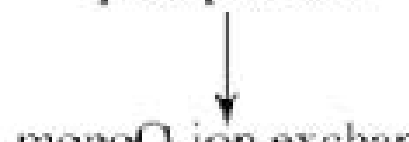
monoQ-ion exchange chromotography

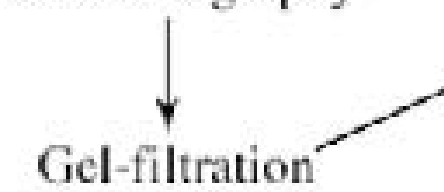
chromotography<smiles>[3H][V]</smiles>

Hydroxyapatite chromotography
B

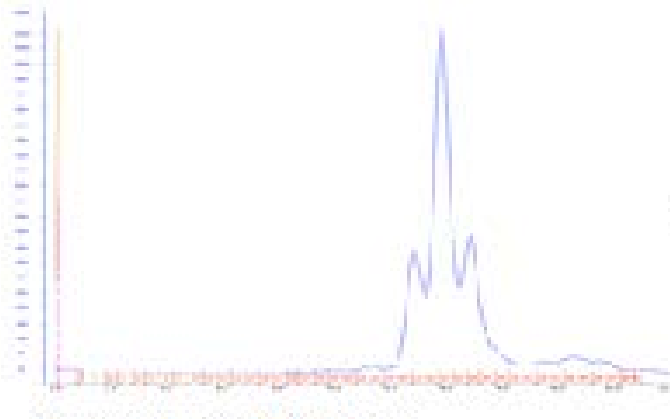

Superdex-200 I'lus

C Fractions after gel-filtration stcp of purification

Westem blot anti-enolase antibodies
TIydroxyapatite 


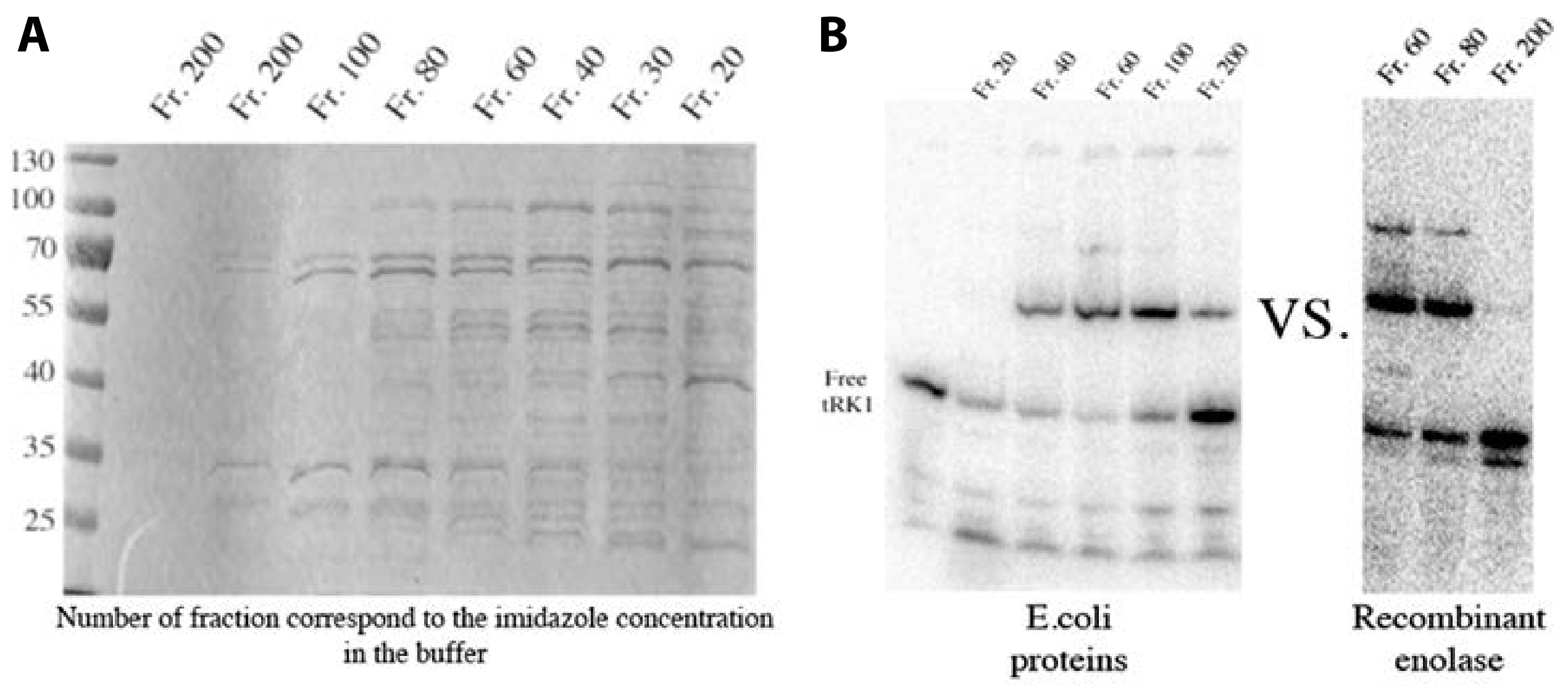




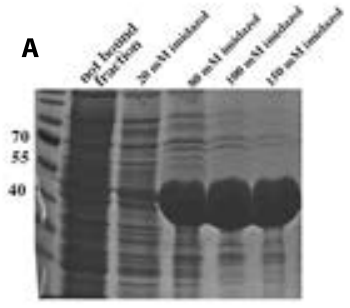

B

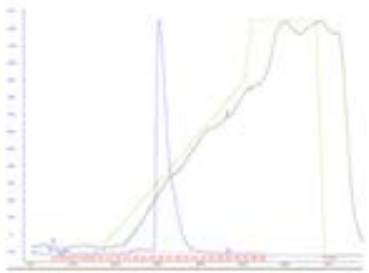

C

D
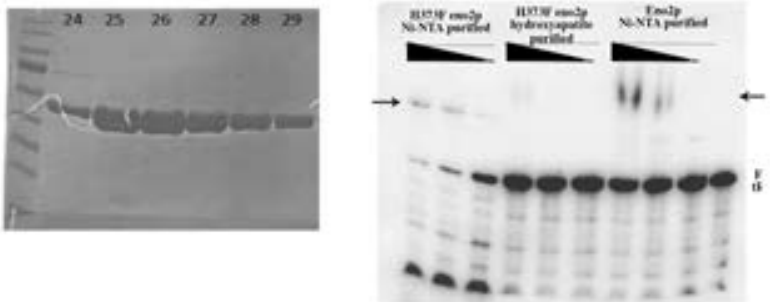
